\title{
UMA AVALIAÇÃO MULTICRITÉRIO DOS RISCOS DO PRÉ-SAL
}

\author{
João Carlos Félix Souza \\ Departamento de Engenharia de Produção \\ Universidade de Brasília \\ João Gabriel Moraes Souza \\ Centro Interdisciplinar de Estudos em Transportes, CEFTRU \\ Universidade de Brasília \\ Simone Borges Simão Monteiro \\ Departamento de Engenharia de Produção \\ Universidade de Brasília \\ Edgard Costa Oliveira \\ Faculdade de Engenharia do Gama \\ Universidade de Brasília
}

\begin{abstract}
Resumo
Identificar os riscos da camada de petróleo, conhecida como Pré-sal, é de extrema importância para o processo de exploração. Alguns desafios do Pré-sal estão relacionados com a profundidade de sua localização e ambiente de exploração inóspito no qual demanda alta tecnologia e conhecimentos específicos. Esse artigo apresenta uma tipologia de riscos associados ao Pré-sal classificados como risco jurídico ou legal, político, militar, econômico, ambiental, financeiro e operacional. Foi realizado um pré-teste com oito grupos de alunos, professores do curso de Engenharia de Produção da Universidade de Brasília, especialistas em gestão de riscos, executivos e pessoas comuns, com o intuito de posteriormente adequar essa pesquisa a outros segmentos de especialistas da área do Pré-Sal. A pesquisa quantitativa utilizou como técnica, para coleta de dados, questionários que foram aplicados para todos os grupos. O objetivo da pesquisa foi ranquear os riscos do Pré-sal, em ordem de importância, a luz da Norma ISO 31000. Destacam-se, como de maior importância, para os grupos pesquisados, os riscos político, legal e operacional e de menor importância o risco militar. Os resultados mostraram a utilidade da ferramenta MCDA/AHP para a ponderação e mapeamento dos riscos para este fim.
\end{abstract}

Palavras-chave: Gestão de Risco, Método AHP, Petróleo, Riscos do Pré-sal.

\section{A Multi-criteria Assessment of the risks of the pre-salt}

\begin{abstract}
Is of extreme importance to the process of exploration identify the risks of the layer of oil, known as pre-salt. Some challenges of the pre-salt layer are related with the depth of its localization and the high technology and the specific knowledge of corrosive environment in which it is inserted. This article presents a typology of risks associated with the pre-salt identified as risk legal, political, military, economic, environmental, financial and operational. A pre-test was performed with eight groups of students, teachers of the Production Engineering course of the University of Brasilia, specialists in risk management, executives and ordinary people; with the intention of adapt later this research to Pre-Salt risks specialists. The quantitative research used as a technique for data collection questionnaires that were applied to all the groups. The objective of the research was to rank the risks of pre-salt in order of importance, considering the norm ISO 31000. This order is one of the most important in that political, legal and operational risk until the minor as the military risk. The results showed the usefulness of the tool MCDA/AHP for the weighting and risk mapping for this purpose.
\end{abstract}

Key-words: Risk management, AHP method, Oil, Risks of pre-salt. 


\section{Introdução}

A gestão de riscos é um conjunto de atividades coordenadas voltadas para o alcance dos objetivos de um grupo social, uma organização, um país. O risco, para a norma 31000 (ABNT, 2009), é o "efeito da incerteza nos objetivos". As atividades do processo de gestão de riscos são gerenciadas para se ter controle sobre os riscos, com o intuito de tratar e reduzir os riscos indesejados, assim como criar oportunidades de segurança e sucesso para alcance dos objetivos.

De acordo com a Figura 1, dentre as atividades do processo de gestão de riscos, segundo a norma ISO 31000, temos a comunicação e consulta, monitoramento e análise crítica, o estabelecimento de contexto, o processo de avaliação de riscos e o tratamento de riscos. O processo de avaliação de riscos contém as atividades de identificação, análise e avaliação de riscos.

Figura 1: Processo de gestão de riscos (ABNT, 2009)

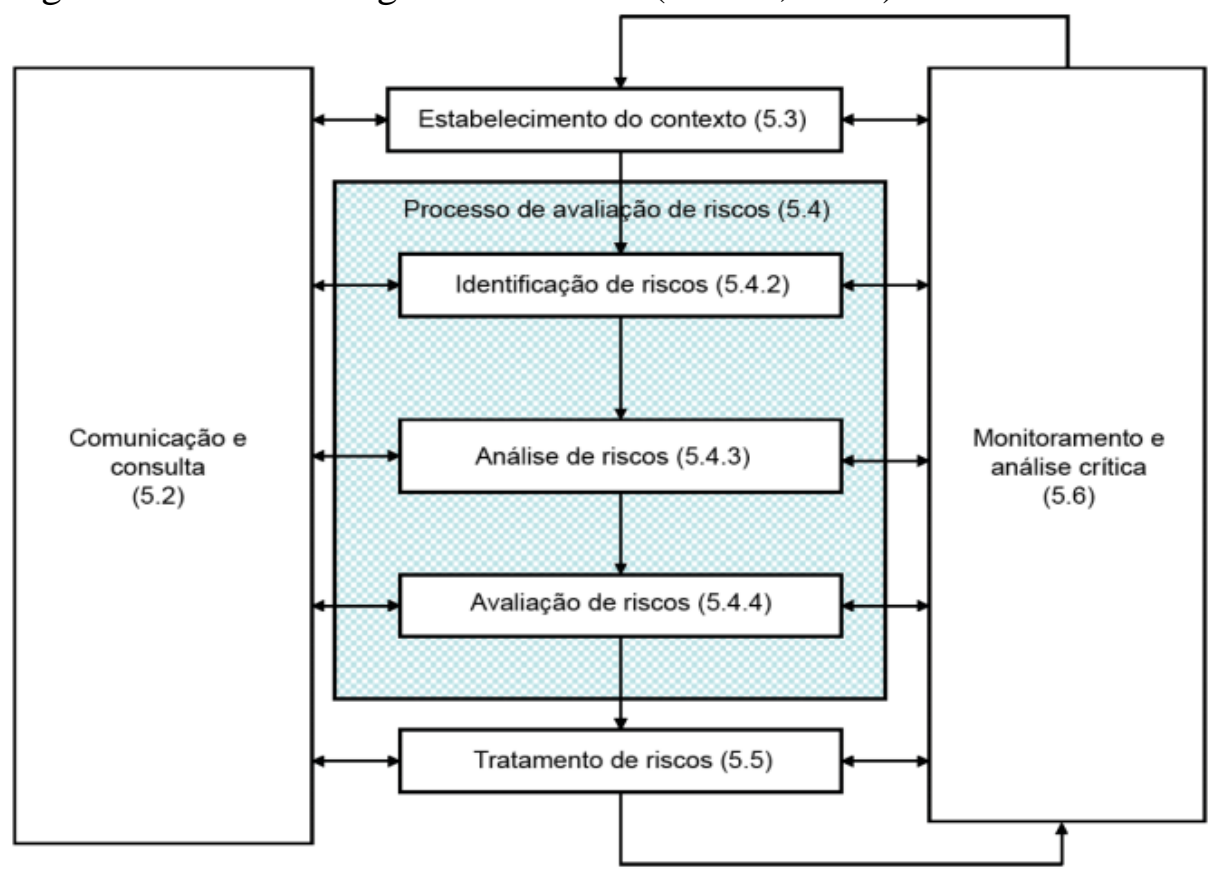

Fonte: relatório da norma ISO 31010

Ao se estabelecer o contexto da gestão de riscos, definem-se tanto os objetivos que se pretende alcançar, quanto os objetos alvos das análises de riscos ou ativos, assim como os critérios de avaliação dos riscos que serão usados nas análises. A identificação dos riscos parte da definição de quais ameaças e vulnerabilidades existe no contexto em análise, respectivamente nos alvos, sejam locais, pessoas, tecnologias, processos, para que o risco possa ser analisado.

A análise de riscos visa à compreensão da natureza do risco para se determinar o nível de risco existente. Ela permite que as ameaças e vulnerabilidades sejam buscadas e, se encontradas, tenham então a probabilidade e o impacto identificados por meio das partes interessadas (ABNT, 2009). O resultado da análise de riscos é avaliar as vulnerabilidades mediante o critério de riscos definido. A análise, para ser efetiva, necessita de uma sistematização quanto ao processo de percepção dos riscos.

A literatura internacional da gestão de riscos, consolidada recentemente em normas da família 31000, versa sobre técnicas para o processo de avaliação de riscos, que foram editadas na recém-lançada norma ISO 31010 (ISO, 2009). A avaliação permite contrastar o resultado 
da análise de riscos com os critérios de riscos estabelecidos, para determinar o grau de tolerância ou aceitabilidade do risco.

A percepção do risco se dá por intermédio da avaliação e a análise de riscos, as quais podem ser feitas a partir de diversas técnicas existentes, sendo de escolha do gestor de riscos a que melhor se adequar ao contexto e complexidade da situação. Tanto podem ser escolhidas técnicas formais como Delphi, HAZOP, AHP, Monte Carlo, como pode simplesmente ser por meio de coleta de evidências, fotografias, descrição de cenários, entrevistas, dentre outros (ISO, 2009). No caso de entrevistas, busca-se a percepção dos riscos por parte das pessoas, organizações envolvidas ou pelas partes interessadas, o fundamental é que deve ter como base o envolvimento da pessoa entrevistada com o assunto em questão.

As pesquisas de opinião, por exemplo, baseiam-se em percepções individuais sobre determinados assuntos. Na gestão de riscos, é importante que as análises tenham como base testemunhos de pessoas tecnicamente envolvidas com o risco, seja sob o enfoque do problema que gera o risco, seja sob o enfoque do controle que trata o risco para que ele seja gerenciado, reduzido e/ou eliminado.

Este trabalho, na segunda seção, aborda alguns conceitos de Análise Multicritério e a metodologia AHP (Analytic Hierarchy Process). A seguir apresenta-se a metodologia utilizada para aplicação da Análise Multicritério nos macrocritérios mapeados para o modelo de risco do projeto Pré-sal. Na terceira seção definem-se os macroprocessos e a metodologia aplicada. A quarta seção aborda a conceituação de risco utilizada para aplicação da pesquisa e contextualização dos participantes. A quinta seção analisam-se os resultados dessa aplicação e finalmente na sexta seção apresentam-se algumas considerações e conclusão.

\section{Análise Multicritério (MCDA)}

Segundo Kou et al (2010), a metodologia MCDA (Multiple Criteria Decision Analysis) utiliza métodos que auxiliam na tomada da melhor decisão diante de múltiplos critérios que envolvem um determinado problema. Ela consiste na estruturação e ponderação de critérios que podem ser utilizados como uma ferramenta de suporte aos tomadores de decisão. Dentre os diversos métodos utilizados pela MCDA, destaca-se o AHP (Analytic Hierarchy Process), ou processo analítico hierárquico, que foi escolhido como o método utilizado na priorização dos riscos deste trabalho. Vargas (1989) define o AHP como uma técnica estruturada para analisar e apoiar tomadas de decisões complexas. Meirelles e Gomes (2009) utilizam a Análise Multicritério em Gestão do Conhecimento na indústria de refino de petróleo. Para mitigação do Risco os autores demonstram a agilidade do processo decisório e, no resultado da aplicação, mostram, através do método, a possibilidade do decisor de transformar um conhecimento tácito em explícito.

O método AHP consiste em três etapas: decomposição dos critérios em uma estrutura hierárquica; comparação entre os critérios de mesmo nível; e conversão dos valores comparativos em valores numéricos normalizados.

Construiu-se uma Estrutura Analítica de Riscos com o intuito de representar os contextos de gestão de riscos que envolvem a exploração da camada Pré-sal no Brasil. Essa estrutura apresenta os objetivos a serem alcançados, os quais foram utilizados para definir os critérios necessários, não, a princípio, para solucionar um problema ou tomar uma decisão, mas para avaliar a percepção de risco de uma possível situação indesejada. Desse modo, o presente trabalho utiliza as técnicas de decisão multicritério para sua resolução. A metodologia de análise multicritério pode ser utilizada em diversas áreas e pretende-se estendê-la para o uso de análise de critérios de priorização de riscos. Os passos adotados podem ser resumidos em: a) definição de pesos para os critérios e b) normalização (Ramos, 2000). 


\subsection{Definição de pesos para os critérios}

Ramos (2000) coloca que não há um método consensual para a definição de pesos, mas várias propostas de procedimentos para este efeito que podem ser encontradas na literatura (Von Winterfeltdt \& Edwards, 1986; Malczewski, 1999).

É possível agrupar os métodos de definição de pesos em quatro categorias: métodos baseados em ordenação de critérios (Stillwell et al., 1981), em escalas de pontos (Osgood et al., 1957), em distribuição de pontos (Easton, 1973) e comparação de critérios par a par (Saaty, 1977). O método aplicado neste trabalho é o de comparação par a par no contexto do processo de tomada de decisão denominado $A H P$. Esta técnica baseia-se numa matriz quadrada $n \times n$, onde as linhas e colunas correspondem aos $n$ critérios analisados no problema. Assim, o valor $a_{i j}$ representa a importância relativa do critério da linha $i$ face ao critério da coluna $j$. Como esta matriz é recíproca, apenas a metade triangular inferior necessita ser avaliada, já que a outra metade deriva desta e a diagonal principal assume valores iguais a 1.

Tabela 1 - Escala para avaliação de riscos (adaptada da escala de Saaty, 1980)

\begin{tabular}{|l|l|}
\hline 1 & O risco é igual ao subcritério $X$ \\
\hline 3 & O risco é um pouco maior que o subcritério $X$ \\
\hline 5 & O risco é moderadamente maior que o subcritério $X$ \\
\hline 7 & O risco é bem maior que o subcritério $X$ \\
\hline 9 & O risco é extremamente maior que o subcritério $X$ \\
\hline
\end{tabular}

Fonte: autores

O estabelecimento de comparações par a par para todos os critérios necessita da definição de uma escala, destinada à normalização, no caso a escala adaptada de Saaty (1980). Os valores 2, 4, 6 e 8 são aceitos e representam opiniões intermediárias às da Tabela 1.

\subsection{Normalização de critérios}

Este processo permite que valores de critérios não comparáveis entre si sejam normalizados para uma mesma escala, viabilizando a agregação entre eles. A maior parte dos processos de normalização utiliza o valor máximo e mínimo para a definição de uma escala. A forma mais simples é uma variação linear definida pela Equação (1) de Eastman (1997), onde $R i$ é o valor a ser normalizado; Rmin o valor mínimo para o critério; e Rmax o valor máximo para o critério.

$$
X i=(R i-R \min ) /(R \max -R \min )
$$

O processo de normalização é, na sua essência, idêntico ao processo de fuzzification introduzido pela lógica fuzzy, segundo o qual um conjunto de valores pode ser expresso ( ou convertido) numa escala normalizada (por exemplo, entre zero e um), tornando-os comparáveis. Para este processo existem várias funções fuzzy que podem ser utilizadas, sendo as mais conhecidas: sigmoidal, $j$-shaped, linear e complexa (Zadeh, 1965).

\section{Metodologia}

Um teste foi realizado com oito grupos de indivíduos, com intuito de observar a percepção de risco sobre o Pré-sal. Os grupos foram divididos em: um grupo de especialistas e pesquisadores em Risco (5 indivíduos), um grupo de executivos e não pesquisadores da área de Risco ( 2 indivíduos), um grupo de alunos de universidade envolvidos em projetos de avaliação de Risco (8 alunos), um grupo de alunos não envolvido em projetos de risco (11 
alunos), porém, contextualizados com textos específicos sobre o assunto e quatro grupos de pessoas comuns (número de participantes de: $3,3,2,2$ ) não envolvidas no assunto de risco. Destaca-se que nenhum grupo está envolvido, diretamente, com pesquisas sobre o Pré-sal. Os valores considerados para o modelo AHP para os grupos de alunos, bem como de pessoas comuns foi em consenso. Para os especialistas e pesquisadores retirou-se a moda do grupo após um teste de Kolmogorov-Smirnov de uma amostra o qual apresentou resultados em que atesta a diferença significativa para a pontuação de cada definição de risco da Figura 2.

A figura 2 ilustra a Estrutura Analítica de Riscos que foi construída com o objetivo de organizar o contexto e/ou áreas potenciais para a gestão de riscos, no nível 1, que podem impactar o desenvolvimento e condução do projeto Pré-sal no Brasil. Portanto, um objetivo específico desse trabalho é identificar as áreas com maior risco para a realização no desenvolvimento do Projeto. O nível 1 (critério de decisão) abrange as áreas de risco legal, risco político, risco militar, risco econômico, risco ambiental, risco financeiro e risco operacional.

Figura 2 - EAR - Estrutura Analítica de Riscos: Pré-sal

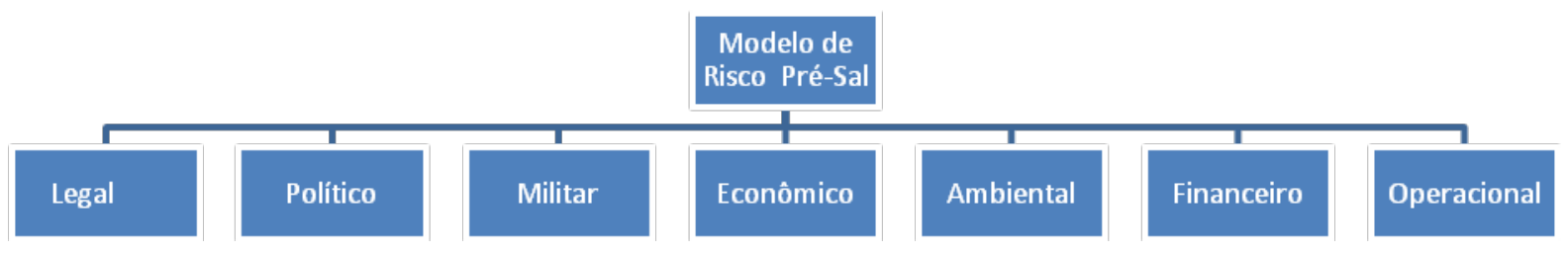

Fonte: autores

Uma pesquisa com trinta e cinco questões foi feita, associando situações de risco das categorias descritas, para que o respondente ou entrevistado pontue numa escala de 1 a 5 (escala de Likert). A intenção é de averiguar, com o método de regressão linear e utilizando como variável dummy, o impacto e a possibilidade de ocorrência de determinada categoria de risco no projeto. A utilização da regressão tem por objetivo atestar o desempenho dos resultados do método multicritério, comparando-os, bem como a coerência nas respostas dos entrevistados.

A avaliação dos riscos foi dividida em duas partes. A primeira consistiu na realização de uma pesquisa com os grupos avaliando e comparando cada uma das categorias ou critérios definidos (risco legal ou jurídico, político, militar, econômico, ambiental, financeiro e operacional) para a identificação da ordem de classificação dos maiores riscos. Para a definição dos critérios em termos da percepção de risco, foi utilizado o método AHP, na qual foi adaptada a escala de Saaty para a elaboração dos questionários. A definição dos riscos (Tabela 2) foi feita mediante o mapeamento de riscos identificados Figura 2.

Tabela 2 - Riscos por critério

\begin{tabular}{|l|l|}
\hline \multicolumn{1}{|c|}{ Critérios } & \multicolumn{1}{|c|}{ Risco } \\
\hline Legal & $\begin{array}{l}\text { Quebra ou inexistência de regulamentos definidos pelas agências } \\
\text { reguladoras e cumprimento de contratos. }\end{array}$ \\
\hline Político & $\begin{array}{l}\text { Interferência político partidária nas empresas responsáveis: Petrobrás e } \\
\text { Pré-sal. Desentendimentos políticos nas distribuições dos Royalties. }\end{array}$ \\
\hline Militar & Incapacidade de defesa das 200 milhas marítimas e, consequentemente, \\
\hline
\end{tabular}




\begin{tabular}{|l|l|}
\hline & das fronteiras onde as reservas estão posicionadas. \\
\hline Econômico & $\begin{array}{l}\text { "Doença Holandesa”. Acomodação dos demais setores produtivos devido } \\
\text { à influência e riqueza facilitada da cadeia produtiva petrolífera. }\end{array}$ \\
\hline Ambiental & $\begin{array}{l}\text { Poluição do mar na extração do petróleo e poluição no transporte, refino e } \\
\text { utilização dos derivados do petróleo. Energias alternativas e } \\
\text { comprovadamente menos poluentes e ecologicamente sustentáveis. }\end{array}$ \\
\hline Financeiro & $\begin{array}{l}\text { Mercado de ativos e opções, bem como influência de desajustes } \\
\text { macroeconômicos, principalmente cambiais. }\end{array}$ \\
\hline Operacional & $\begin{array}{l}\text { Barreiras tecnológicas (profundeza dos poços), logística, tributária e de } \\
\text { qualificação de mão de obra. }\end{array}$ \\
\hline
\end{tabular}

Fonte: autores

A segunda parte consistiu na análise de probabilidade de ocorrência dos riscos versus impacto com avaliação de questionário com perguntas que indicavam situações de risco associadas as sete categorias definidas acima. Os entrevistados atribuíam uma nota (numa escala de Likert de 1 a 5, onde 5 representa o evento de maior probabilidade de risco versus impacto conforme sua percepção quanto ao risco àquela situação (Tabela 3).

Tabela 3 - Escala de probabilidade e impacto

\begin{tabular}{|c|c|c|}
\hline Escala & Probabilidade & Impacto \\
\hline $\mathbf{1}$ & $\begin{array}{c}\text { Probabilidade muito baixa de } \\
\text { ocorrência (até 20\%) }\end{array}$ & $\begin{array}{c}\text { Impacto muito pequeno na } \\
\text { realização do evento }\end{array}$ \\
\hline $\mathbf{2}$ & $\begin{array}{c}\text { Probabilidade baixa de } \\
\text { ocorrência (até 40\%) }\end{array}$ & $\begin{array}{c}\text { Impacto pequeno na } \\
\text { realização do evento }\end{array}$ \\
\hline $\mathbf{3}$ & $\begin{array}{c}\text { Probabilidade média de } \\
\text { ocorrência (até 60\%) }\end{array}$ & $\begin{array}{c}\text { Impacto médio na realização } \\
\text { do evento }\end{array}$ \\
\hline $\mathbf{4}$ & $\begin{array}{c}\text { Probabilidade alta de } \\
\text { ocorrência (até 80\%) }\end{array}$ & $\begin{array}{c}\text { Impacto alto na realização do } \\
\text { evento }\end{array}$ \\
\hline $\mathbf{5}$ & $\begin{array}{c}\text { Probabilidade muito alta de } \\
\text { ocorrência (no mímino 80\%) }\end{array}$ & $\begin{array}{c}\text { Impacto muito alto na } \\
\text { realização do evento }\end{array}$ \\
\hline
\end{tabular}

Fonte: autores, baseado na escala de Likert

\subsection{Riscos do Pré-sal}

Cada um dos critérios de decisão do nível 1 (Figura 2 e Tabela 2 anteriores) pode ser compreendido por definições mais detalhadas de risco apresentadas aos pesquisadores e pesquisados, as quais fizeram parte da contextualização de entendimento no sentido de compreenderem melhor o arcabouço jurídico, econômico, financeiro e operacional para o projeto Pré-sal. Entende-se que essa contextualização foi fundamental para se ter um conhecimento mínimo, a fim de obter resultados coerentes e consistentes. Porém, não se pretende fazer uma explanação profunda e detalhada de cada critério de risco definido, não é o foco deste trabalho. Estas particularidades foram identificadas para facilitar o entrevistado e entrevistador nos conceitos que se desejam pesquisar.

\section{Resultados}

No primeiro momento da aplicação do método foram analisados os riscos em cada um dos critérios associados (risco jurídico, político, militar, econômico, ambiental, financeiro e 
risco operacional). Dos oito grupos analisados apenas três apresentaram resultados consistentes na análise multicritério, são eles: o grupo de pesquisadores especialistas em Risco (chamaremos de grupo1), o grupo de alunos que trabalham com avaliação de risco (grupo 2) e o grupo de alunos que foram contextualizados com material específico do assunto de risco (grupo 3). O teste de consistência analisa a coerência das respostas, isto é, alguns entrevistados, por exemplo, podem priorizar o risco jurídico ao ambiental e este ao político. No entanto, priorizam o político ao legal o que traduz uma incoerência. O resultado nesse caso resulta em inconsistência que aqui não analisaremos. Esses grupos, como descrito anteriormente, não foram consistentes nas respostas pela metodologia AHP, bem como nas respostas para o modelo de Regressão. O teste $F, R$ múltiplo e os testes $t$ student para validação do impacto de cada categoria de risco foi inconclusivo.

A tabela 4 mostra os resultados da pesquisa aplicados com base no método AHP para a identificação da percepção dos maiores riscos, neste caso os maiores vetores prioridade encontrados.

As matrizes de comparação do modelo AHP utilizado apresentam em suas linhas e colunas os critérios que foram comparados, seguindo a lógica de comparação do elemento da linha para com o elemento da coluna, conforme a escala de Saaty adaptada apresentada na tabela 1. Na última coluna da tabela, temos o Vetor Prioridade (w) que indica o peso do critério em relação aos demais comparados, neste caso o valor percentual dos maiores riscos encontrados para cada tipo de risco. Ressalta-se que os resultados apresentados são das matrizes que tiveram a sua consistência devidamente verificada, o que garante a qualidade dos resultados.

Tabela 4 - Matriz de comparação dos critérios de Risco do Pré-sal - Grupo 1

\begin{tabular}{|c|r|}
\hline RISCO & Vetor Prioridade $(w)$ \\
\hline Legal & $18 \%$ \\
\hline Político & $25 \%$ \\
\hline Militar & $5 \%$ \\
\hline Econômico & $7 \%$ \\
\hline Ambiental & $12 \%$ \\
\hline Financeiro & $10 \%$ \\
\hline Operacional & $23 \%$ \\
\hline Total & $100 \%$ \\
\hline
\end{tabular}

Fonte: autores

A tabela 4 mostra que a percepção dos especialistas de gestão de risco (grupo 1) sobre maiores riscos para o critério Risco Político, Operacional, Legal, Ambiental, Financeiro, Econômico e Militar, respectivamente.

$\mathrm{Na}$ análise de regressão só foram significativos, em testes de hipóteses, as respostas referentes aos riscos Operacionais, Político e Legal, nesta ordem de importância. O modelo no teste $F$ foi significativo e o $R$ múltiplo de 0,73 . Portanto, em termos de resultado da percepção de risco com o grupo 1 os resultados das metodologias foram semelhantes e coerentes. A tabela 5 apresenta a matriz de comparação dos critérios do grupo 2.

Tabela 5 - Matriz de comparação dos critérios do Grupo 2

\begin{tabular}{|l|r|}
\hline RISCO & Vetor Prioridade (w) \\
\hline Legal & $3 \%$ \\
\hline
\end{tabular}




\begin{tabular}{|l|r|}
\hline Político & $20 \%$ \\
\hline Militar & $23 \%$ \\
\hline Econômico & $12 \%$ \\
\hline Ambiental & $3 \%$ \\
\hline Financeiro & $8 \%$ \\
\hline Operacional & $31 \%$ \\
\hline Total & $100 \%$ \\
\hline
\end{tabular}

Fonte: autores

Segundo a tabela 5, os maiores riscos para o Grupo 2 encontram-se, respectivamente, no risco operacional, militar e político. Quanto à análise de regressão os resultados significativos foram apenas os referentes aos riscos operacional, militar e legal. $\mathrm{O}$ fato de o critério de risco legal ter aparecido na análise de regressão como item importante, muito embora, no modelo multicritério não o tenha, pode ter origem na possível avaliação como risco operacional nesta análise de regressão. Os problemas jurídicos de regulação e trabalhistas muitas vezes, na literatura específica, são categorizados como riscos operacionais. O modelo, neste caso, foi significativo em $F$ e $R$ múltiplo de 0,54 .

A tabelas 6 apresenta as demais matrizes de comparação para os critérios do Grupo 3 :

Tabela 6 - Matriz de comparação dos critérios do Grupo 3

\begin{tabular}{|l|r|}
\hline RISCO & Vetor Prioridade (w) \\
\hline Legal & $21 \%$ \\
\hline Político & $11 \%$ \\
\hline Militar & $3 \%$ \\
\hline Econômico & $17 \%$ \\
\hline Ambiental & $4 \%$ \\
\hline Financeiro & $5 \%$ \\
\hline Operacional & $39 \%$ \\
\hline Total & $100 \%$ \\
\hline
\end{tabular}

Fonte: autores

Conforme a tabela 6 percebe-se que a ordem de prioridade de risco é do Operacional, Jurídico, Econômico, Político e os demais sem importância relativa. Quanto à regressão destacou-se apenas o fator jurídico ou risco legal como um fator significativo para o impacto na percepção de risco dos entrevistados.

Após a obtenção dos resultados, a Estrutura Analítica de Riscos (EAR) inicialmente apresentada foi atualizada (gráfico 1 e figura 5), obtendo-se uma visão holística de toda a análise realizada com base na percepção dos riscos segundo o modelo AHP adaptado. Optouse por apresentar apenas o resultado do grupo 1, por dois motivos: o grupo foi composto por especialistas na área de risco e os resultados estão mais aderentes com o modelo de regressão utilizado para comparação.

gráfico1: Resultado da Matriz de comparação de critérios - Grupo 1 


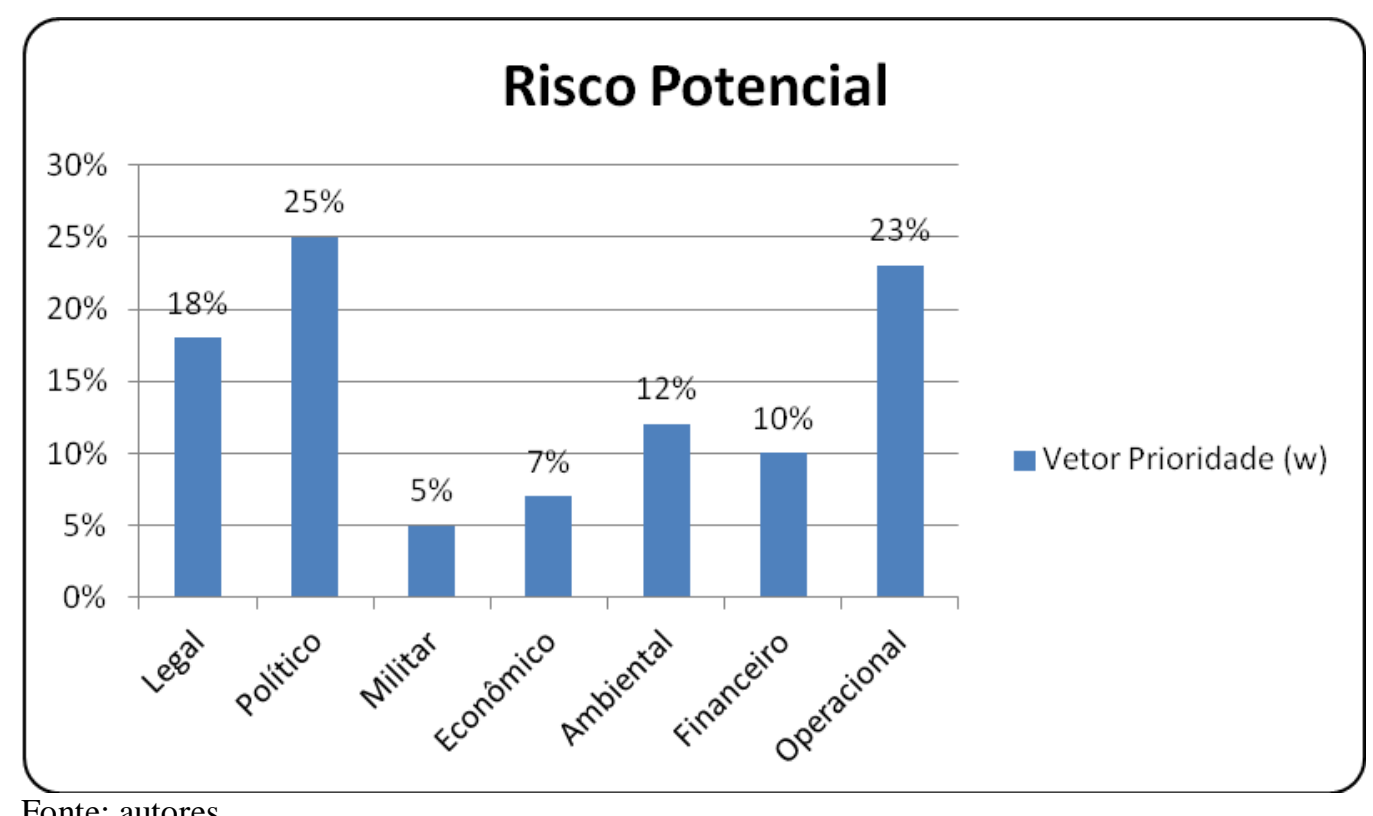

Fonte: autores

Figura 5 - EAR atualizada para o Grupo 1

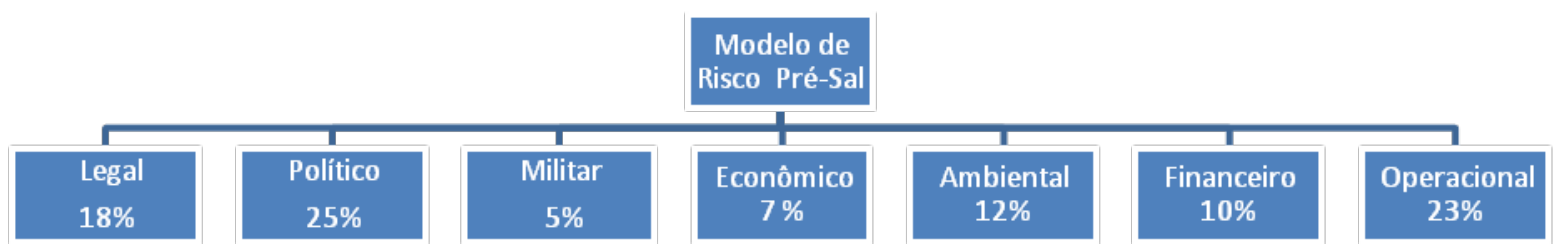

Fonte: autores

\section{Conclusões}

A área de gestão de riscos está em pleno desenvolvimento como ciência. Ainda carece de abordagens teóricas e metodológicas, principalmente no que se refere ao processo de avaliação de riscos.

O trabalho apresentado neste artigo permitiu validar uma ferramenta que pode ser muito útil para a avaliação, monitoramento e mitigação dos riscos no contexto dos riscos inerentes ao projeto do Pré-sal.

A prova de conceito foi utilizada com o objetivo de validar a ferramenta de identificação, análise e avaliação de riscos. Com base nos resultados apresentados, conclui-se que a ferramenta obteve sucesso na identificação da percepção de riscos, no entanto, restrita a uma contextualização por parte dos pesquisadores quanto aos conceitos de risco e suas definições, para que não tenham interpretações heterogêneas. A ferramenta também possibilita que especialistas de várias áreas de risco contribuam com a visão do risco de uma maneira ampla, porém categorizada.

O resultado do trabalho foi o mapeamento por categorias ou critérios de risco à identificação e a gravidade do risco por áreas, cuja percepção define priorização no tratamento e mitigação dos possíveis riscos. Demonstrou que a MCDA e o AHP são muito úteis para a ponderação de riscos e sua automatização. Por meio de tabelas pode facilitar a 
identificação, análise e avaliação dos riscos que precisam ser gerenciados para sucesso no projeto. Neste detalhe, e avaliando o resultado, cabe observar com mais cuidado a preocupação quanto ao risco Político e as influências políticas para o projeto que se desenvolve na camada Pré-Sal, se não é o maior problema, em geral, é aquele que mais preocupa os entrevistados (gráfico 1). Isto explica um pouco o cuidado que os autores tiveram de esmiuçar e esgotar as explicações políticas e, principalmente, legais e jurídicas contratuais envolvidas no tema, visto que estas são resultados exaustivos de negociações políticas.

Como sugestões para trabalhos futuros propõem-se uma aplicação da ferramenta para outros grupos de gestores e profissionais envolvidos direta ou indiretamente nas demais áreas analisadas e no planejamento do projeto como um todo, buscando uma maior avaliação de riscos baseada na percepção de riscos dos profissionais e responsáveis pelo Pré-Sal. Ademais, a ferramenta deve permitir, em sua evolução, a inclusão de dados factuais sobre o andamento da realização do projeto.

\section{Referências}

ASSOCIAÇÃO BRASILEIRA DE NORMAS TÉCNICAS. NBR ISO/IEC 31000: Gestão de Riscos - Princípios e Diretrizes, 2009.

DAMODARAN, A. Avaliação de Investimentos. Ferramentas e Técnicas para a Determinação do Valor de Qualquer Ativo. 2a. Edição ampliada. Qualitymark. Rio de Janeiro, 2010.

DAMODARAN, A. Gestão Estratégica do Risco. Uma referência para tomada de riscos empresariais. Wharton School Publishing. Bookman. Porto Alegre, 2009.

EASTMAN, J.R. IDRISI for Windows: User's Guide. Version 2.0. Clark University Graduate School of Geography, Worcester, MA, USA, 1997.

EASTON, A. Complex Managerial Decision Involving Multiple Objectives. John Wiley \& Sons, New York, NY, USA, 1973.

ISO/IEC 31010. Risk management - Risk assessment techniques, 2009.

ISO Guia 73. Gestão de Riscos: vocabulário, 2009.

KOU, G.; SHI, Y.; WANG, S. Multiple criteria decision making and decision support systems - Guest editor's introduction, (c) 2010 Elsevier B.V. All rights reserved.

MEIRELLES, C.L.A.; GOMES, L.F.A.M. O apoio Multicritério à decisão como instrumento de Gestão do Conhecimento: Uma aplicação á Indústria de Refino de Petróleo. Revista Pesquisa Operacional v.29 n.2 pp 451-470, maio a agosto de 2009.

MINISTÉRIO DAS MINAS E ENERGIA. Modelo Regulatório do Pré-Sal 2013. http://www.mme.gov.br/mme/menu/pre_sal.html.

MINISTÉRIO DAS MINAS E ENERGIA. Projeto de Lei número 5.938/2009. http://www.mme.gov.br/mme/galerias/arquivos/pre_sal/Projeto_de_Lei_5938.pdf.

NEPOMUCENO, F. \& SUSLICK, S.B. Alocação de recursos financeiros em projetos de risco na exploração de petróleo. RAE - Revista de Administração de Empresas, 40(1), 63-75, São Paulo, jan/mar 2000.

OSGOOD, C.E.; SUCI, G.J. \& TANNENBAUM, P.H. The Measurement of Meaning. University of Illinois Press, Urbana, IL, USA, 1957. 
RAMOS, R.A.R. Localização Industrial - Um Modelo Espacial para o Noroeste de Portugal. Braga - Portugal. 299p. Tese (Doutorado), Universidade do Minho, 2000.

SAATY, T.L. A Scaling Method for Priorities in Hierarchical Structures. Journal of Mathematical Psychology, 15(3), 234-281, 1977.

SAATY, T.L. Analytical Hierarchy Process: Planning, Priority Setting, Resource Allocation. MCGraw-Hill, New York, NY, USA, 1980.

SAATY, T.L. \& VARGAS, L.G. Prediction, Projection and Forecasting. Kluwer Academic Publishers, Boston, MA, USA, 1991.

SERRA, R.V. \& FERNANDES, A.C. A distribuição dos Royalties Petrolíferos no Brasil e os riscos de sua "Financeirização". RDE - Revista de Desenvolvimento Econômico, Salvador (BA). Janeiro, 2005.

STILLWELL, W.G.; SEAVER, D.A. \& EDWARDS, W. A Comparison of Weight Approximation Techniques in Multiattribute Utility Decision Making. Organization Behavior and Human Performance, 28(1), 62-77, 1981.

VARGAS L. G. An overview of the Analytic Hierarchy Process and its applications. Joseph M. Katz Graduate School of Business, University of Pittsburgh, Pittsburgh, PA 15260, USA. November 1989.

VON WINTERFELDT, D. \& EDWARDS, W. Decision Analysis and Behavioural Research. Cambridge University Press, Cambridge, UK, 1986.

YAGER, R.R. On Ordered Weighted Averaging Aggregation Operators in Multicriteria Decision Making. IEEE Transactions on Systems, Man and Cybernetics, 8(1), 183-190, 1988.

ZADEH, L.A. Fuzzy Sets. Information and Control, 8, 338-353. Zambon, K. L.; Carneiro, A A F M; Silva, A N R; Negri, J. C. (2005). Análise de Decisão Multicritério na Localização de Usinas Termoelétricas utilizando SIG. Pesquisa Operacional, v.25 n.2 p183-199 maio-agosto, 1965. 